\title{
Spectrum Handoff Aware AODV Routing Protocol for Cognitive Radio Vehicular Ad Hoc Networks
}

\author{
Abdur Rashid Sangi (D), ${ }^{1}$ Mohammed S Alkatheiri, ${ }^{2}$ Satish Anamalamudi, ${ }^{3}$ \\ Mohammed A. Alqarni, ${ }^{4}$ Muhammad Hammad Memon, ${ }^{1}$ and Wanan Yang ${ }^{1}$ \\ ${ }^{1}$ School of Artificial Intelligence and Big Data, Yibin University, Yibin 644000, Sichuan, China \\ ${ }^{2}$ Department of Cybersecurity, College of Computer Science \& Engineering, University of Jeddah, Jeddah, Saudi Arabia \\ ${ }^{3}$ Department of Computer Science Engineering, SRM University, Amaravati, Andhra Pradesh, India \\ ${ }^{4}$ Department of Software Engineering, College of Computer Science and Engineering, University of Jeddah, Jeddah, Saudi Arabia
}

Correspondence should be addressed to Abdur Rashid Sangi; sangi_bahrian@yahoo.com

Received 24 May 2021; Revised 11 July 2021; Accepted 13 August 2021; Published 15 September 2021

Academic Editor: Yue Song

Copyright ( $\odot 2021$ Abdur Rashid Sangi et al. This is an open access article distributed under the Creative Commons Attribution License, which permits unrestricted use, distribution, and reproduction in any medium, provided the original work is properly cited.

\begin{abstract}
End-to-end application performance and throughput of vehicular cognitive transport control protocol depend on how efficiently the segments (TCP header) are being transmitted from source to destination. One way to enhance the performance of vehicular cognitive TCP protocol is by reducing the packet drops in between the source and destination. In general, packet drops occur in between the source and destination of Cognitive Radio Vehicular Ad Hoc Network (CR-VANET) because of spectrum handoff or cognitive node handoff, or network congestion. In this paper, we focus on enhancing the performance of cognitive TCP protocol through "cognitive AODV routing protocol with the spectrum handoff mechanism." In the proposed work, channel-route control messages of cognitive AODV routing protocol are updated with the support of spectrum handoff which helps to provide the backup opportunistic channel during PU active and helps to reduce the end-to-end spectrum handoff packet drops. Simulation results reveal that the overall performance of the vehicular cognitive TCP protocol with the proposed spectrum handoff aware cognitive AODV routing protocol is enhanced as compared to the existing cognitive TCP protocol.
\end{abstract}

\section{Introduction}

With enhanced wireless technology, a wide range of applications are being deployed and operated in current static unlicensed ISM spectrum bands. This results in spectrum scarcity and increased spectral congestion. On the contrary, FCC (Federal Communication Commission) revealed that $15 \%$ to $85 \%$ in the current static allocated spectrum bands below $3 \mathrm{GHz}$ are not efficiently utilized concerning temporal and spatial domains [1-4]. This clearly shows that the current static spectrum allocation is not utilized optimally rather than physical spectrum scarcity. One way to overcome this issue is by utilizing the temporal and spatial domain unused spectrum bands through dynamic spectrum access. With dynamic spectrum access (DSA), the existing spectrum scarcity problem will be alleviated as well as it increases the spectrum utilization. These result in developing an adaptive wireless device called cognitive radio together with the introduction of dynamic spectrum access through secondary spectrum licensing called "CRN (Cognitive Radio Networking)." With CRN, cognitive radio devices are capable of accessing the spatial spectrum dynamically through spectrum holes or white spaces [5-7]. Moreover, a CR node with "SDR (Software Defined Radio)" is capable of switching in a wide range of spectrum bands (spectrum hole) through available RSSI (signal strength), transmission quality, and dynamic PU activity. In general, Cognitive Radio Networks can be deployed in one of the two architectures, namely, "infrastructure-based CRN" and "ad-hoc CRN networks," which are also called "CRAHNs (Cognitive Radio Ad Hoc Networks)" [8-12]. Recent advancement of wireless applications in vehicular networks leads to spectrum scarcity $[4,5]$ which can be overcome through the CR-VANETs (Cognitive Radio Vehicular Ad Hoc Networks). 
This paper mainly focuses on the performance enhancement of "vehicular CRAHNS" by reducing the packet drops due to spectrum handoff and mobile handoff of the vehicular nodes. Currently, the main research focus in cognitive radio networking is well explored in the areas of channel estimation, spectrum sensing, spectrum sharing, cognitive routing, and MAC (medium access control). In general, the list of PU-free channels in between one-hop CR nodes will be managed by the "connection management" [13-15]. During the route discovery at the network layer, the routing protocol has to concurrently select the PU-free channel (spectrum hole) and next-hop route to transmit the application data. Thus, there should be tight coordination between "connection management" and "routing tables" during the end-to-end channel-route discovery. In [16], the design of cognitive AODV routing protocol is being proposed to concurrently select the channel along with the route during the route discovery. Later, an end-to-end channel route is identified by exchanging the cognitive control message with a "common control channel" for application data transmission. In static spectrum-allocation-based mobile ad-hoc networks, the application data packets in between the source to destination CR node can be dropped due to bandwidth reduction or mobile handoff. When it comes to Cognitive Radio Ad Hoc Networks, there is a new type of packet drop that occurs because of spectrum handoff from the current spectrum hole to another spectrum hole at the time of PU active in the current CR communication channel [17-19]. State-of-the-art research proposals are proposed and designed to reduce the packet drops due to CR node handoff and bandwidth degradation. But, very few research activities are done to explore the packet buffering mechanisms during spectrum handoff. When it comes to dynamic spectrum access, it is crucial to reduce the application packet drops during spectrum handover to achieve enhanced end-to-end application throughput. To accomplish this, this paper proposes a "packet buffering and forwarding mechanism" to the "cognitive AODV routing protocol." With this, the endto-end achievable TCP throughput will be enhanced by reducing on-the-fly packet drops and reducing the channel-route reconstruction time during spectrum handover at the intermediate CR node. Our paper is organized as follows. Section 2 briefly explains the advantages and disadvantages of the existing CRAHNs without handoff mechanisms. Section 3 describes the proposed "spectrum handoff" aware cognitive AODV protocol through packet buffering and forwarding mechanism to enhance the performance of end-to-end connection-oriented cognitive TCP protocol. Besides, a RED (Random Early Detection) buffer is proposed to use in our solution to minimize the packet drops during packet forwarding in between intermediate CR nodes. Section 4 explains the simulation results collected by simulating in the Cognitive Radio Network Simulator (CRNS.2.35) and compares the performance of existing cognitive TCP protocols with proposed simulation results with spectrum handoff packet buffering and forwarding mechanism. Section 5 discusses the conclusion with future work.

\section{Motivation}

In standalone wired or wireless networks, application packet drops are mainly due to increased traffic flow (buffer overflow) that results in network congested packet drops. In addition, packet drops may occur at the end nodes or intermediate nodes due to errors within the packet. Extensive research is carried out to minimize the packet drops due to buffer overflow and errors in the packet. Later on, once the mobility support is deployed within the wireless networks, then node mobility packet drops are increased during node handover from one base station to another base station. To overcome this, many researchers focus on enhancing the performance of the end-to-end packet transmission by introducing the node mobility handover mechanisms. But, the node mobility handover mechanisms cannot be directly deployed in cognitive radio vehicular networks due to opportunistic channel utilization and high-speed node mobility. Hence, a vehicular node should aware of the opportunistic PU-free channel along with the node mobility to integrate the spectrum handover and node mobility in real-time vehicular communication. To achieve this, our work proposes a spectrum handoff-aware routing protocol that considers robust dynamic PU channel selection along with node mobility support at the time of discovering the end-to-end channel route from the source to destination vehicular nodes.

\section{Related Work}

The end-to-end connection-oriented application throughput mainly depends on the reliability of the channel routes that are being discovered through the routing protocol. Hence, the cognitive routing protocol in the network layer plays a critical role in enhancing the end-to-end throughput of the cognitive TCP protocol.

In general, MAC protocols are broadly classified into contention-based (random access) and contention-free access protocols to schedule the one-hop data transmission. Traditional random access-based MAC protocols make use of CSMA/CA for accessing the one-hop channel whereas MAC contention-free protocols use either predetermined global or local time-slotted-based channel access. With CSMA/CA, mobile nodes at the MAC layer will contend for the shared channel (nonoverlapping channel) to transmit the application data, whereas virtual carrier sensing with NAV (Network Allocation Vector) helps to avoid the nodes' hidden terminal problem at the sensing range. Random access-based MAC protocols are further classified into RTS/ CTS-based and tone-based [6] to alleviate the hidden and exposed terminal issues. But, when it comes to cognitive radio networks, there is no single global unlicensed channel (2.4 GHz/5 GHz ISM bands) available to assign as a control channel for cognitive control message exchange. To overcome this, in [20-22], the authors proposed a hybrid CCCbased MAC and routing protocols for channel-route control message exchange at the network layer and one-hop scheduling operations at the MAC layer of Cognitive Radio Ad Hoc Networks. An overall summarization of well-known routing protocols is shown in Table 1 .

The comparison was made with different state-of-the art routing protocols in VANET and Cognitive Radio Ad Hoc Networks. To date, most of the existing solutions focus on 
TABLE 1: Summarization of routing protocols.

\begin{tabular}{lcccc}
\hline Routing protocol & Routing metric & Channel selection & Energy conservation & CCC \\
\hline OBPF [23] & Link quality & Single channel & No & No \\
SE-AOMDV [24] & Hop count & Single nonoverlapping channel & No & Yes \\
CR-AODV [16] & Optimized channel route & Single channel & Yes & Dedicated \\
ERMR [25] & Energy and stability & Channel availability time & No & Dedicated \\
MRPC [26] & Hop count & Stability & No & Dedicated \\
PMRC [27] & Route path & Single channel & & \\
\hline
\end{tabular}

channel-route discovery at the network layer for application data transmission. But, there is little research focused on how to handle the spectrum handover during application data transmission. Besides, end-to-end packet delays in cognitive routing protocol and CR node energy consumption mainly depend on the PU activity (spectrum handoff), per-hop channel saturation, and CR node handoff. Moreover, the number of simultaneous noninterfering transmissions among the neighbor $\mathrm{CR}$ nodes will have a direct impact on achievable end-to-end network throughput in multihop cognitive communication [28-31]. Furthermore, CCC (Common Control Channel) [20,32] plays a vital role in discovering the end-to-end channel-route path for application data transmission. In common mobile IP-based networks, the end-to-end path failure can be mainly due to node mobility from one subnetwork to another subnetwork. When it comes to Cognitive Radio Ad Hoc Networks, a new path failure in between the source to destination CR node can happen due to spectrum handoff in PU transmission channels. As shown in Figure 1, whenever the PU (primary user) is active in the current CR node communication channel, then on-the-fly packets have to be buffered until a new one-hop channel route is being locally discovered. Cutting-edge routing protocols are being proposed to provide the shortest end-to-end channel route between the source and destination CR node. But, whenever there is a spectrum handoff, there is no existing solution to handle onthe-fly packet drops until a new local channel route is being reconstructed. Also, there should be a tight synchronization between the network layer and the spectrum management to check the common PU-free channels available between the intermediate CR nodes. Apart from this, when the intermediate CR node is unable to find the backup PU-free channel at the time of spectrum handoff, then it generates the channel RERR (route-error) message and also unicasts back to the source CR node.

Once the RERR message arrives back at the Source CR node, then it will re-initiate the channel-route discovery through Route-Request and Route-Response to handle the spectrum handover connection failure. An enormous amount of research is well focusing on solution designing for channel route failures due to the spectrum handover with efficient PU-free back up the channel to continue the ongoing transmission. In $[21,22]$, the authors mainly focus on the PU channel selection at the gateway devices to integrate multiple unlicensed radio access networks. But, the node mobility is not integrated with PU channel selection which results in packet drops during the active primary user in the ongoing CR vehicular communication. In [29, 30], the authors focus on ways to enhance PU-free channel utilization due to the proliferation of radio devices and the heterogeneous environment of wireless communications. In their contribution, the SU channel switching rate is considered when the primary user is active in the current CR communication. But, there is no mechanism explored about how the SU channel switching has to be integrated along with the node mobility and the traffic characteristics to minimize the application packet drops. To date, there is no research focus on how to reduce on-the-fly packet drops through packet buffering and packet forwarding mechanisms during cognitive channel switching and CR node mobility. In this paper, a RED-based packet buffering and forwarding mechanism is being proposed to minimize onthe-fly packet drops to enhance the performance of cognitive TCP protocol with respect to spectrum mobility and CR node mobility. The main contribution of this paper is threefold: (1) packet buffering and forwarding mechanism to buffer on-the-fly spectrum handover packet drops, (2) modified CR-AODV protocol to record the PCL (PU-free channel list) during the channel-route discovery, and (3) mechanism to trigger the spectrum management with the available PU-free channel list to buffer the packets during spectrum handover.

\section{The Proposed Scheme}

Spectrum handoff-aware cognitive AODV routing protocol works under the half-duplex radio transceiver, i.e., a secondary radio device can either receive or transmit at any given time through the opportunistic PU-free spectrum bands. In traditional IEEE 802.11 ad-hoc networks, the network designer has to mainly focus on how to efficiently utilize the nonoverlapping spectrum in either $2.4 \mathrm{GHz}$ or $5 \mathrm{GHz}$ ISM bands. Thus, a PLRR (Preemptive Local Route Repair) is being proposed to locally reconstruct the link during end-to-end application data transmission. With this, the achievable TCP throughput in traditional ad-hoc networks can be improved by locally re-constructing the routes. Initially, when a sender/source node has application data, then it has to check for the end-to-end channel route. If the channel route is unavailable within the routing table of the sender/source CR node, then it has to initiate the RREQ broadcast message.

Since there is no fixed static channel available in Cognitive Radio Ad Hoc Networks, it is a challenging task to broadcast the route discovery control messages from the source to destination CR node. In other words, with decentralized cognitive ad-hoc radio networks, it is clear that 


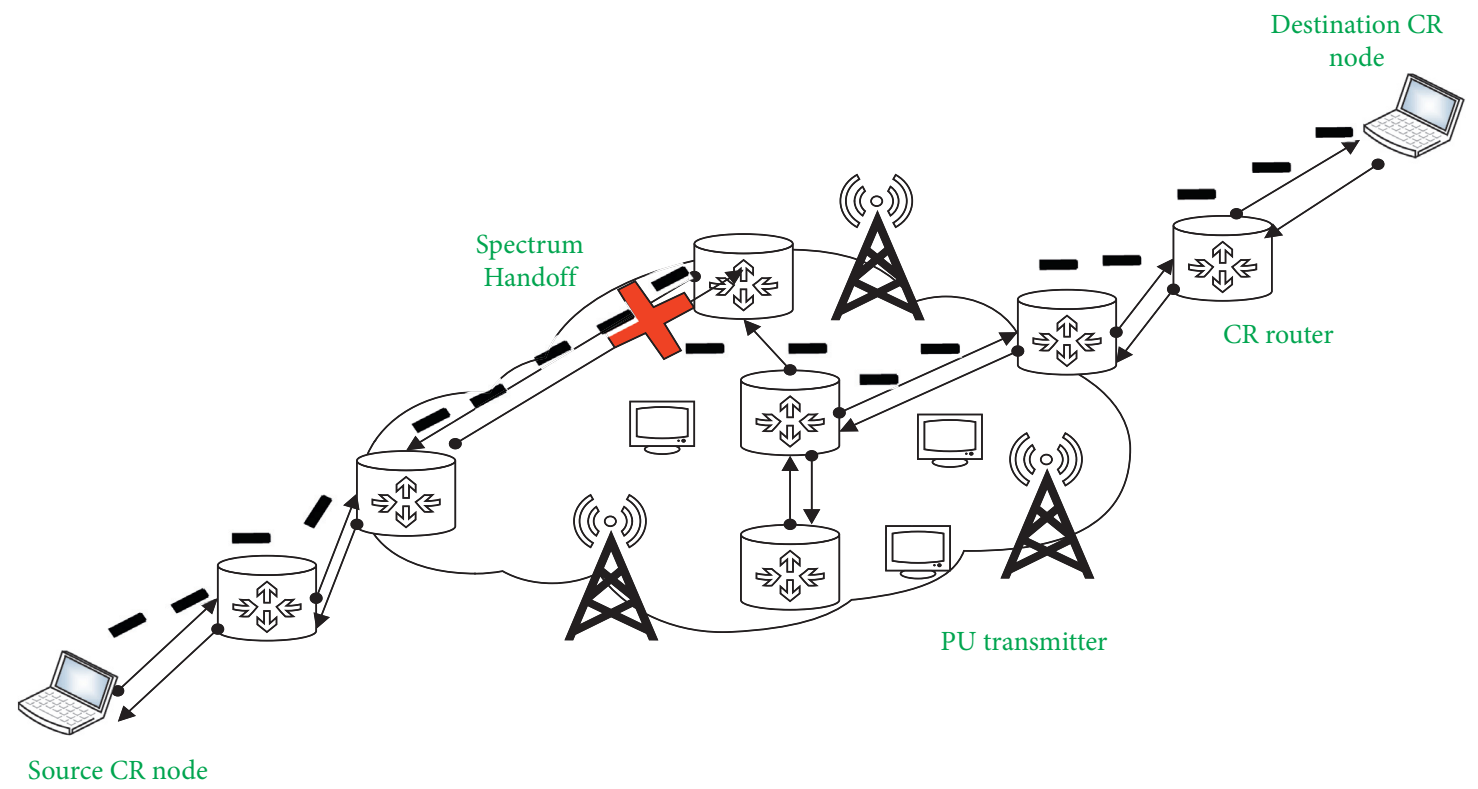

FIGURE 1: Spectrum handoff in between intermediate CR nodes due to PU active.

any CR node synchronization with other nearby CR nodes for cognitive control exchange is a huge challenge. Moreover, the AODV routing protocol with dynamic spectrum access has certain limitations to select the end-to-end channel-route in between the source and destination CR node. This is due to increased route-channel control overhead, longer channel rendezvous end-to-end delays, and increased collision probability because of the multichannel hidden terminal issue. Hence, hybrid CCC (Common Control Channel) is being proposed to transmit the cognitive and route control messages among source and destination CR nodes. As shown in Figure 2, hybrid CCC makes use of $902 \mathrm{MHz}$ ISM band for Time " $T-1$ " to exchange its PU-free channel list to one-hop neighbor CR nodes. Subsequently, at $T-2$, the source $\mathrm{CR}$ node will start listening to its PU-free channel list to learn about the PU-free channel list of its one-hop away neighbor nodes. With this, the sender/source CR node will get the PCL list of its one-hop away neighbor CR nodes. Later, the source CR node broadcasts the DCRequest in a common PU-free channel to its one-hop away neighbor nodes. CR nodes that have the same PU-free channel will reply with the DCResponse back to the source CR node. With this, the source CR node will have a synchronized common PU-free channel available with its one-hop neighbor CR nodes. Thus, the routing and MAC control messages (RREQ, RTS, CTS, and RREP) will be exchanged in between the source and destination to find out an end-to-end shortest path to transmit the application data. As shown in Figure 2, packet drops can occur at the intermediate CR node due to PU active in the ongoing CR node communication channel. At this point, the per-node packet buffering technique can be used to avoid the application packet drops. This will, in turn, enhance the achievable application throughput at the cognitive-TCP protocol. In this paper, omnidirectional antenna-based simulation is being used for both cognitive control exchange through CCF (Cognitive Coordination Function) and application data transmission through DCF (Distributed Coordination Function), as shown in Figure 2. If the end-toend channel route does not exist at the source CR node, then it will activate the medium access layer to initiate the CSMA/ CA (Carrier Sense Multiple Access with Collision Avoidance) for one-hop link-level channel contention to broadcast the channel RREQ (route-discovery messages). Figure 3 shows how the packet buffering mechanism works in our proposed work.

On-the-fly packets will be recorded at the SDR radio interface and buffered at the input RED buffer. When there is no issue with the outgoing link, then the buffered packet in the input buffer will be sent to the output RED buffer. Whenever there is a link failure at the outgoing RED buffer towards the next-hop CR node, then a separate RED packet buffer will be created for the next-hop neighbor CR node. In this paper, spectrum handover in-flight packets from the old CR intermediate node to a new CR intermediate node have to be forwarded through this priority-based RED buffer. At one point in time, RED buffer will have either Avg_quelength $<$ Thresoldmin or Thresoldmin $<$ Avg_quelength $<$ Thresoldmax, or Avg_quelength $>$ Thresoldmax. In mobile ad-hoc networks, a separate packet buffering for mobile handover will be created when the average length of the input RED buffer is in between Minimum_Thresold to Maximum_Thresold or when the average length is greater than the Maximum_Thresold. But for spectrum handover packet drops, a separate spectrum handover buffer will be created for in-flight packets, when the old CR intermediate node handovers its opportunistic channel due to PU active. Thus, the received in-flight spectrum handover packets at the old CR node will be buffered at the spectrum handover packet buffer. It is noteworthy that a separate spectrum 


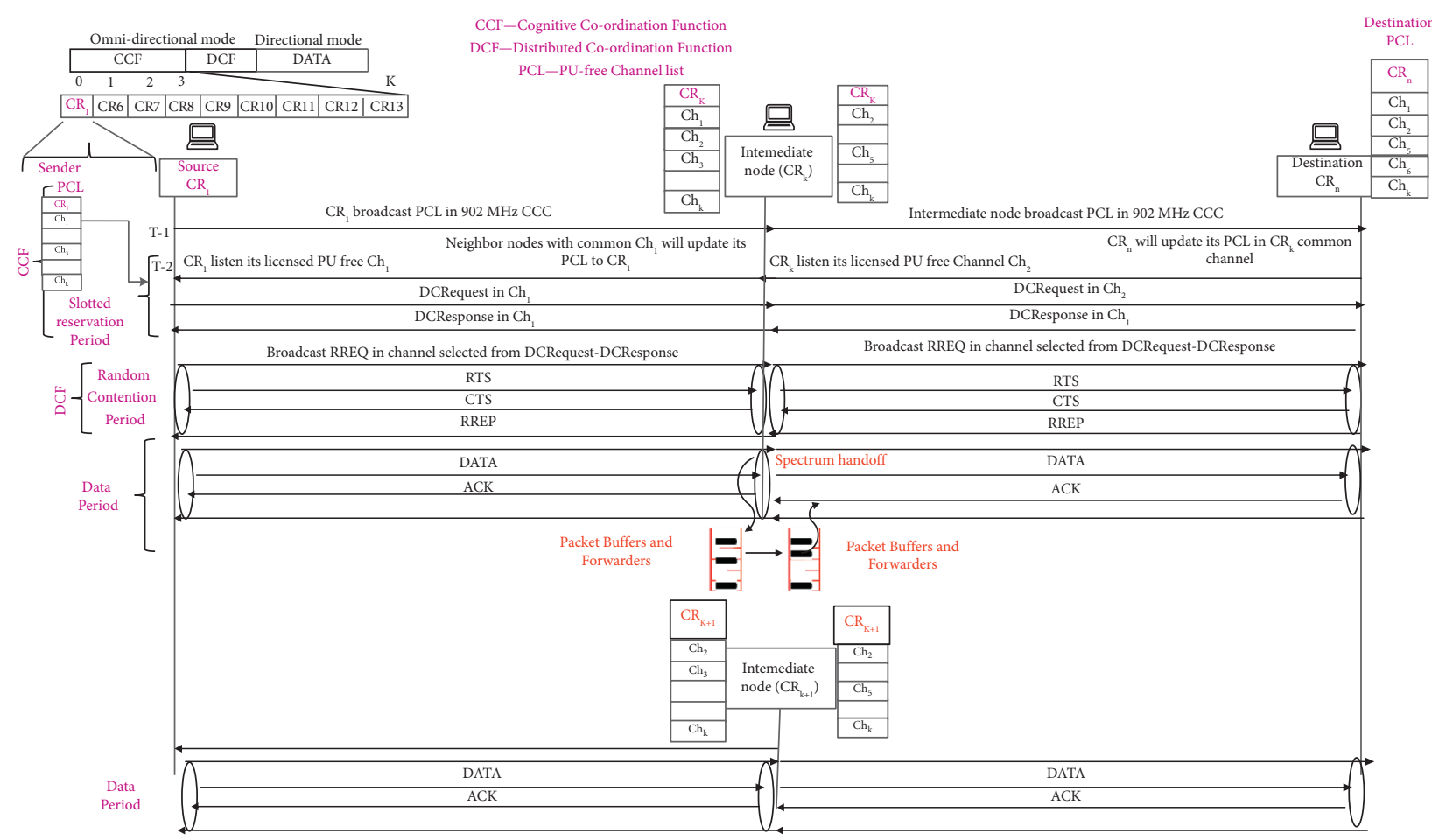

FIgURE 2: Hybrid CCC-based CR-AODV protocol with spectrum handoff through packet buffering and forwarding.

handover packet buffer will be created for every next-hop CR node that has spectrum handover link failure.

Algorithm 1 explains the packet buffering operation when the average length of the RED buffer is less than the predefined threshold value. Once there is a spectrum handover, then the old CR node will check for the local channel-link re-construction through PLRR (Preemptive Local Route Repair). Later, spectrum handover buffer packets will be retransmitted from the spectrum handover buffer to the next-hop CR node (new CR node) through the outgoing RED buffer. In this case, once the link is locally reconstructed, then the spectrum handover buffer packets are transmitted like normal best-effort IP traffic to the nexthop CR node (new CR node). But there are cases where the RED buffers can get overflow through network congestion. This usually happens due to the store-and-forward mechanism of multiple traffic flows. In this case, whenever there is network congestion, then the outgoing RED buffer will start dropping the packets randomly at the output RED buffer. This in turn results in spectrum handover packet drops even with the packet buffers. To overcome this, a priority-based spectrum packet buffering and forwarding mechanism is being proposed to avoid the spectrum handover packet drops during the network congestion at the output RED buffer. Figure 4 describes how to add a priority option to the spectrum handover buffer packets at the output RED buffer. As shown in Figure 4, a one-bit priority will be added to the spectrum handover packet whenever the average length of the output RED buffer is either Thresoldmin $<$ Avg_que_length $<$ Thresoldmax or Avg_quelength $>$ Thresoldmax. At this point in time, priority packets (spectrum handover packets enabled with priority bit $P=1$ ) cannot be discarded at the output RED buffer of the old CR node during the network congestion. Whenever the average length of the output RED buffer at the old CR node is Avg_quelength $<$ Thresoldmin, then the priority bit is disabled (priority bit $P=0$ ) and packets will be transmitted like normal IP packets. With this, the probability of spectrum handover buffer packet drops will be reduced especially when there is network congestion due to increased besteffort traffic flows at the output RED buffer of the old CR node. This helps to engage the opportunistic communication link to transmit many application packets than the routecontrol messages. Thus, the end-to-end application throughput and performance of cognitive TCP protocol with proposed priority packet buffering will be enhanced in comparison with the existing spectrum handover-based packet buffering and forwarding. In the worst case, there may be packet drops at the input RED buffer due to network congestion. Algorithm 2 explains the packet buffering operation when the average length of the RED buffer is in between a minimum threshold and a maximum threshold of a predefined threshold value. For such cases, especially for deterministic applications, a separate priority input buffer will be created along with the normal input buffer at the network layer.

Once the packets with higher priority arrive at the old CR node, then it is buffered within the priority buffer. Later, the CR node checks for the outgoing PU-free channel to forward the buffered priority packet. When there is a spectrum handover, then the buffered priority packet will be forwarded to the spectrum handover buffer and waited until the new one-hop link is established from the current CR node. It is noteworthy that the buffered spectrum handover 


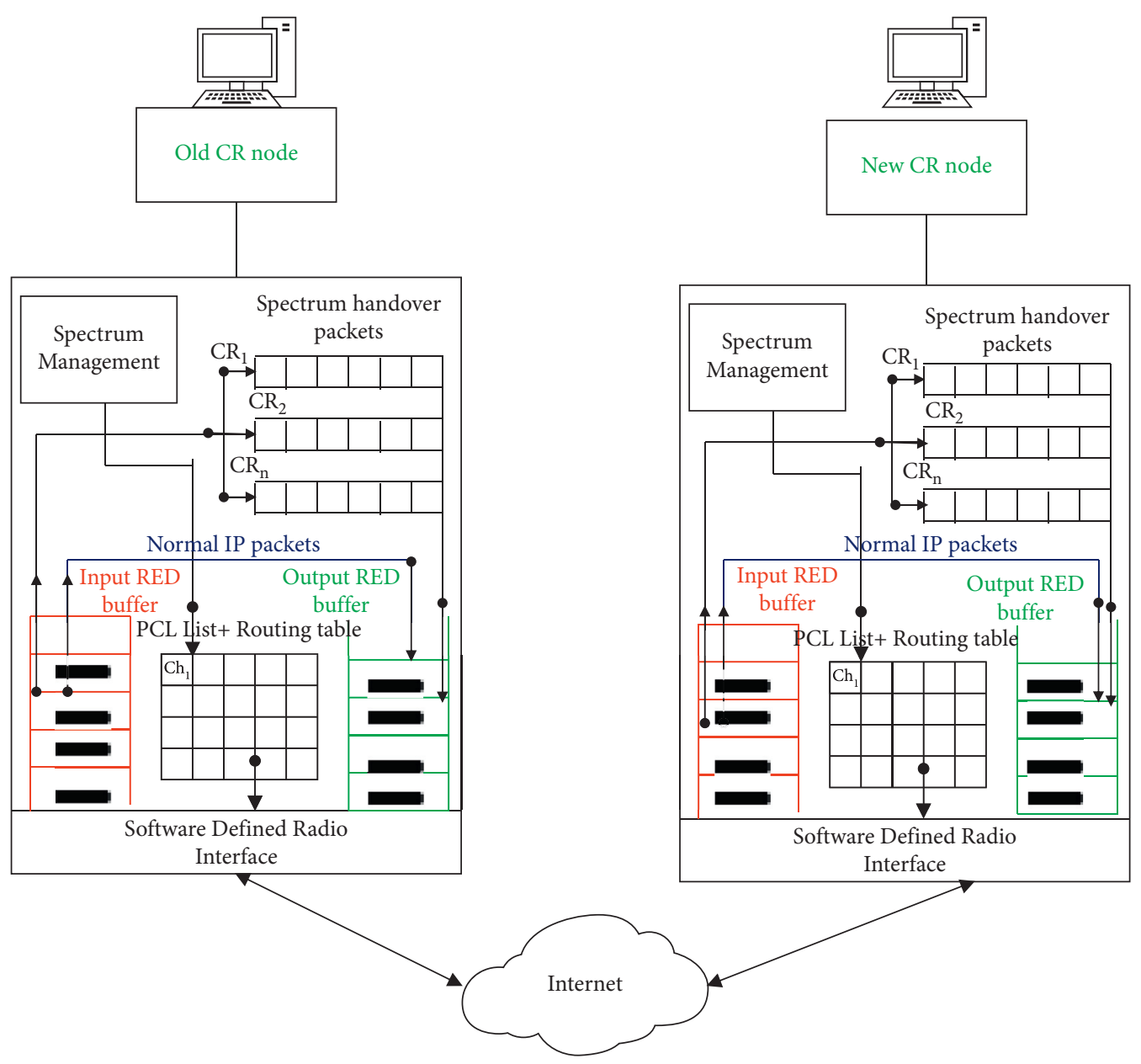

FIGURE 3: Spectrum handoff packet buffering at the intermediate CR node in CRAHNs.

packet will be forwarded with either priority bit as " 1 " or " 0 " based on the average length of the output RED buffer. Once the packet arrives at the next hop intermediate CR node (new CR node), then it will check the status of the input RED buffer and decide whether it has to be buffered in the input RED buffer or priority input buffer.

4.1. End-to-End Channel-Route Maintenance. Due to "dynamic opportunistic spectrum access," there will be frequent link failures due to active licensed primary users. In widely used MANETs (mobile ad-hoc networks), there is a control message called "RERR (Route Error)" that triggers when the outgoing link cannot be locally reconstructed. This usually happens when there is network congestion of mobile handoff in traditional mobile ad-hoc networks. But, in Cognitive Radio Ad Hoc Networks, there is an additional packet drop due to channel switching at the time of PU active. Hence, the RERR (Route Error) message should be updated with the support of the "spectrum handover packet drops" to the source CR node. In [21], the authors described a new control message format to support the spectrum handover packet drops at an intermediate CR node to the sender/source CR node. When the link cannot be locally reconstructed for more than an RTT (Round Trip Time), then this intermediate CR node has to re-initiate the "RERR (Route Error)" message back to the source CR node. Thus, the RERR (Route Error) message with error bit is set to " 11 " at the time of transmitting from the intermediate CR node to the source CR node [21]. Once, the source CR node receives the "Route Error" message with the "Spectrum handover" link failure, then it will start to look for an existing alternate route. When there is no existing alternate route, then it initiates the route discovery message to determine the fresh channel route from source towards destination CR nodes.

4.2. Spectrum Handover Queuing Model. Whenever the average queue length of output RED buffer at intermediate CR node is Avg_Que_length_CR $<$ thresholdmin, then there is no need of having priority bit enabled at the spectrum handover packet buffers. Whenever the average queue length of the output RED buffer is in between Thresoldmin $<$ Avg_que length $<$ Thresoldmax and Avg_quelength $>$ Thresoldmax, then the spectrum handover packet buffers will be enabled with priority bit at the spectrum handover buffers. Hence, it is crucial to know the average length of the output RED buffer through a mathematical model to enable the priority bit for congested output RED buffer. An M/M/1 queuing model was designed to figure out 


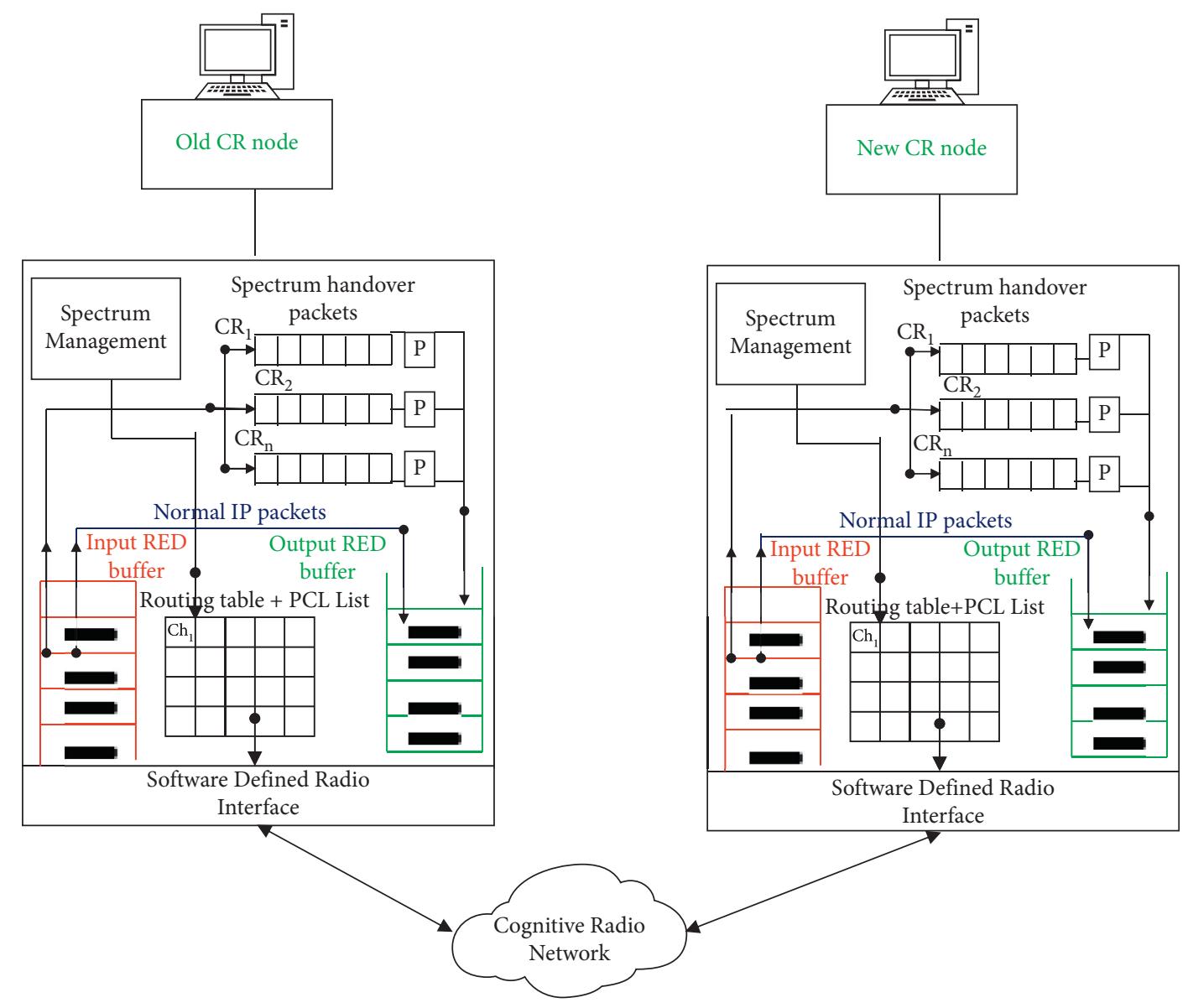

FIgURE 4: Spectrum handoff packet buffering with priority buffers at the intermediate CR node in CRAHNs.

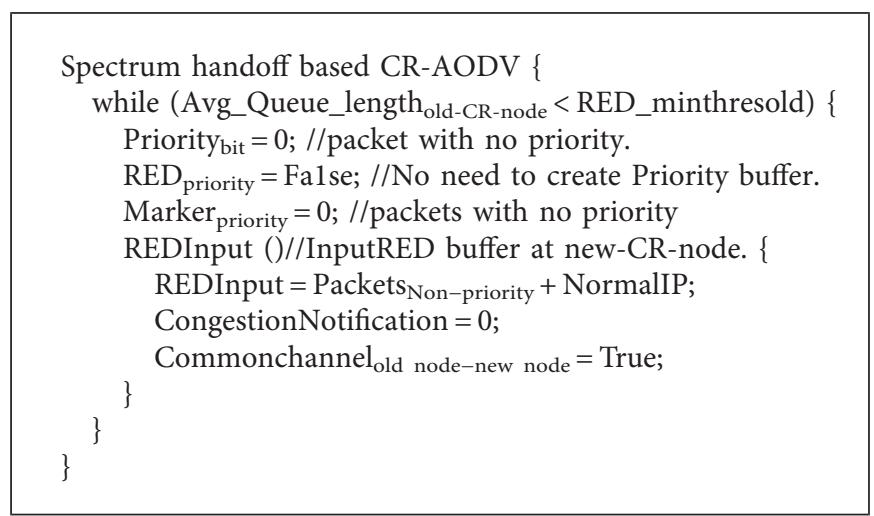

Algorithm 1: //Spectrum handoff packet buffering at intermediate CR nodes//

the average length of the output RED buffer at a certain point in time. Based on the analysis of the mathematical model, a priority bit will be decided to enable/disable at the spectrum handover buffer. In queuing theory, an $\mathrm{M} / \mathrm{M} / 1$ Queue represents the length of the queue in a system having a single server, and the packet arrival is determined by the Poisson process, and the job service times are based on the exponential distribution. The state space in $\mathrm{M} / \mathrm{M} / 1$ queue is the set of $\{0,1,2,3, \ldots$.$\} whose value corresponds to the number$ of receiving packets (normal IP traffic flows and spectrum handover packets) from one intermediate CR node (old CR node) to another intermediate CR node (new CR node). In this paper, the following assumptions are as follows: packet arrival (both spectrum handover + normal IP packets) rate at the old CR node is " $\alpha$ " from state " $P$ " to " $P+1$." Service time is defined as $1 / €$ where " $€$ " is the service rate. The server follows the FCFS (First Come First Serve) basis to receive the packets. When there is no spectrum handover, then the packet leaves the input RED buffer, thus reduced to " $N-1$ " if the total number of the packet is known to be "N." In 


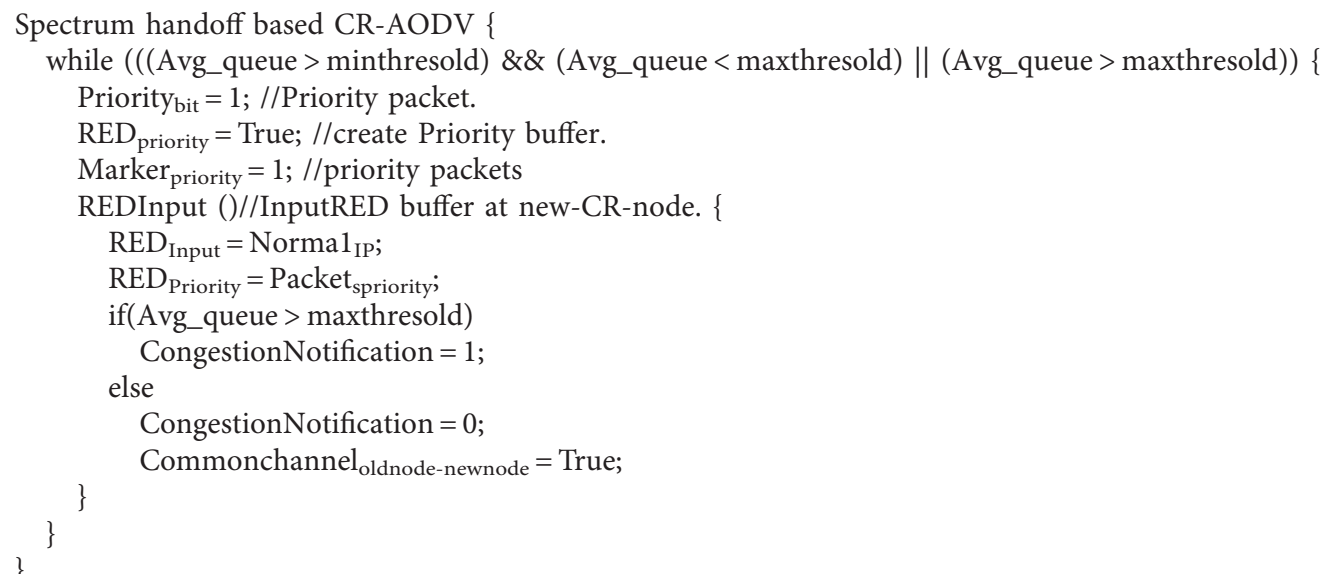

Algorithm 2: //Spectrum handoff packet buffering with priority buffers at intermediate CR nodes//

general, it is assumed that the RED buffer is of finite size $(N)$ which is having a limit on the storage of the packets within the queue. The average length (1) of the proposed queuing model is

$$
\mathrm{Lq}=\sum_{n=0}^{\infty}(n-1) p_{n}=\frac{2 p-p^{2}}{1-p}+\frac{p^{N-1}(N p+1)}{1-p^{n-1}} .
$$

The detailed derivation of "Average Queue Length (Lq)" is explained in [22]. Here, when $P=1$, then $\mathrm{Lq}=0$, i.e., there will not be spectrum handover packet buffering at the input RED buffer. When $P>1$, then Lq is equal to infinity. Hence, we only analyze when $P<1$ to determine the average length of the queue.

\subsection{One-Hop Theoretical Throughput Calculation.} Theoretical upper bound of one-hop throughput from the old CR node to the new CR node helps to determine the collision rate concerning to the data rate in the PU-free opportunistic shared channel. Furthermore, this helps to predict the congestion window size variation at the cognitive transport layer to improve the end-to-end throughput and overall CRAHN network performance. In theoretical throughput estimation, we assume that the channel is completely available for the CR node to transmit the application data. This helps for an ideal case to figure out the maximum link achievable throughput without any collisions. In experimental results, the PU active in current CR communication is considered to show how the throughput and performance of the TCP vary with respect to PU active:

$$
\begin{aligned}
& \text { Throughput }_{\text {per-hop }}=\frac{T_{\text {data }}}{\text { Delay }}, \\
& \text { Throughput }_{\text {per-hop }}=\frac{T_{\text {data }}}{T_{\mathrm{DCF}}+T_{\mathrm{PCL}}},
\end{aligned}
$$

where

$T_{\mathrm{PCL}}=$ Time to select the PU channel,

$$
\begin{aligned}
& T_{\mathrm{DCF}}= T_{\mathrm{RTS}}+T_{\mathrm{SIFS}}+T_{\mathrm{CTS}}+T_{\mathrm{SIFS}}+T_{\mathrm{DATA}}+T_{\mathrm{SIFS}}+T_{\mathrm{ACK}}+T_{\mathrm{DIFS}}+T_{\mathrm{BACKOFF}}, \\
& T_{\mathrm{DCF}}= T_{\mathrm{RTS}}+T_{\mathrm{SIFS}}+T_{\mathrm{CTS}}+T_{\mathrm{SIFS}}+\left(T_{\mathrm{PR}}+T_{\mathrm{PHY}}+\frac{8\left(L_{\mathrm{MAC}}+\mathrm{MSDU}\right)}{\text { Data }_{\text {Rate }}}\right) \\
&+T_{\mathrm{SIFS}}+\left(T_{\mathrm{PR}}+T_{\mathrm{PHY}}+\frac{8\left(L_{\mathrm{MAC}}\right)}{\text { Data }_{\text {Rate }}}\right)+2 * T_{\text {slottime }}+T_{\mathrm{SIFS}}+\frac{C w_{\text {min }} * T_{\text {slottime }}}{2}, \\
& T_{\mathrm{PCL}}= T_{\mathrm{PR}}+T_{\mathrm{PHY}}+\frac{8\left(n * L_{\mathrm{PCL}}+L_{\text {sender IP }}+L_{\text {sender MAC }}\right)}{\text { Data rate }}, \\
& \text { Throughput }_{\text {end-to-end }}=N *\left(\frac{T_{\text {data }}}{\text { Delay }}\right)
\end{aligned}
$$


where $N=$ total number of hops in between the source to destination CR node.

Equation (4) clearly shows that the achievable end-toend application throughput directly depends on the route control messages. Whenever there are spectrum handoff packet drops, then the time taken to reconstruct the channel route increases. Thus, the end-to-end delay on data transmission is higher with increased control message transmission.

\subsection{Use Case of Proposed CR-AODV Protocol in CR Vehicular} Ad Hoc Networks. Packet drops in high-speed vehicular networks, such as automated ambulance, are a significant issue that needs to be addressed in mission-critical applications. In addition, relying on the unlicensed spectrum bands to transmit the application data should also be addressed due to heavy traffic flows by heterogeneous networks in the ISM bands. To overcome both the issues, this paper proposes a cognitive-AODV routing protocol that utilizes an unlicensed spectrum band along with the spectrum mobility and CR vehicular node mobility with RED spectrum mobility priority packet buffers. The proposed cognitive AODV-routing protocol mainly addresses the packet drops with respect to node mobility, spectrum mobility, and aggregate packet drops at the intermediate CR vehicular nodes. The performance of the proposed CR routing protocol is tested with different performance metrics in Sections 5 and 6.

\section{Implementation in NS-2 Simulator}

CR-NS-2.31 (Cognitive Radio Network Simulator) $[18,33-37]$ with dynamic channel access support is implemented to analyze the congestion window variation, performance of cognitive TCP, and end-to-end throughput with respect to spectrum handover (PU active) at the intermediate CR nodes in Cognitive Radio Ad Hoc Networks. This work makes use of the $8 \mathrm{MHz}$ "primary TV white space" for opportunistic data transmission among the source CR node and destination CR node within the CRAHNs. Table 2 below describes the parameters used for our simulation. The total simulation area is considered as $1000 * 1000$ meters. Cognitive Ad-hoc on-demand distance vector (CognitiveAODV) routing protocol is used to select the unreliable endto-end concurrent channel route from the source CR node to the destination CR node. The total number of CR nodes in between the sender CR node to destination CR node is considered as 100 whereas PU nodes are considered as 10 . The cognitive TCP protocol is being used to provide reliable end-to-end connection-oriented traffic flow.

During the simulation, it is assumed that there is no PU transmitter active at the current $\mathrm{CR}$ communication. Once the connection setup (CR-TCP) and end-to-end channelroute discovery (CR-AODV) are done, then application data start to transfer from the sender CR node to the destination CR node. At this point in time, the PU transmitter will be active (PU-ON) for either 10 or 15 , or 20 seconds. Subsequently, the spectrum handover will get triggered at an intermediate CR node, and on-the-fly spectrum handover packets get dropped at an intermediate CR node with the existing CR routing protocols.

\section{Results and Discussions}

With the implementation of spectrum handover-based CRAODV protocol in cognitive network simulator, the packets at the intermediate CR-node get buffered at the spectrum handover buffers and re-transmitted back once the local channel-route is discovered for the spectrum handover link failure. Figure 5(a) describes the CR-TCP congestion window variation with varied simulation times. It is noteworthy that the end-to-end CR throughput and the performance of cognitive-TCP are directly dependent on the congestion window.

Whenever there is a packet drop, then the ACK will not be reached back to the sender TCP within RTT (Round Trip Time). Due to the longer RTT delays, the sender congestion window gets reduced which in turn reflects on the rate of packet transmission. When there is a PU active in current $\mathrm{CR}$ communication, then the intermediate CR node drops the spectrum handover packets due to channel-route failure. In Figure 5(a), CR-AODV without spectrum handover buffer is having more packet drops than the intermediate CR nodes, which is why the size of the congestion window is less in comparison with the other two techniques. CR-AODV with only spectrum handover buffer is having a better congestion window size in comparison with the CR-AODV without spectrum handover buffer. This is due to a separate spectrum handover buffer at an intermediate CR node for the next-hop CR node that has a channel-link failure. CRAODV with spectrum handover buffer and priority bit is having the highest congestion window. This is due to reduced packet drops at the output RED buffer. In other words, whenever the best-effort traffic flow is close to maximun_thresold, then the spectrum handover packets also get dropped along with the normal IP packets. To overcome this, the proposed solution added a priority bit to the spectrum handover buffer packets. With this, the output RED buffer will try to not drop the packet that is having priority bit but will continue to drop the normal IP packets. This results in an increased TCP congestion window in comparison with the other two techniques. Figure 5(b) describes the end-to-end CR-TCP throughput (Mbps) with respect to simulation time. In reality, the end-to-end throughput is directly proportional to the size of the congestion window. With this, cognitive CR-AODV with spectrum handover buffer with priority bit is having better achievable throughput in comparison with CR-AODV with only spectrum handover buffer and CR-AODV without spectrum handover buffer. Figure 5(c) describes the local channel-route discovery with different no. of CR nodes. With a higher number of one-hop CR nodes, there will be increased traffic flow at the input RED buffer and output RED buffer. This results in network congestion and increased packet drops at the input RED buffer and output RED buffer. Since the proposed CR-AODV with spectrum handover buffer is having priority bit enabled, the output 
TABLE 2: Simulation parameters.

\begin{tabular}{lc}
\hline Parameter name & Description \\
\hline Topology & $1000 * 1000$ flatgrid \\
No. of CR nodes & 100 \\
No. of PU channels & $10(8 \mathrm{MHz}$ channels \\
No. of PU transmitters & 10 \\
PU active probability & 10,15, and 20 seconds \\
Mobility model & Random waypoint model \\
Receiver threshold & $-95 \mathrm{dBm}$ \\
Carrier sense threshold & $-115 \mathrm{dBm}$ \\
Input CR transmit power & $10 \mathrm{~mW}$ \\
CR Tx range & $200 \mathrm{~m}$, licensed channel \\
PU Tx range & $500 \mathrm{~m}$, licensed channel \\
Data rate & $2 \mathrm{Mbps}$ \\
Interface queue length & 50 \\
Simulation time (s) & 200 seconds \\
Traffic type & TCP \\
Packet size & 512 and 1024 bytes \\
\hline
\end{tabular}

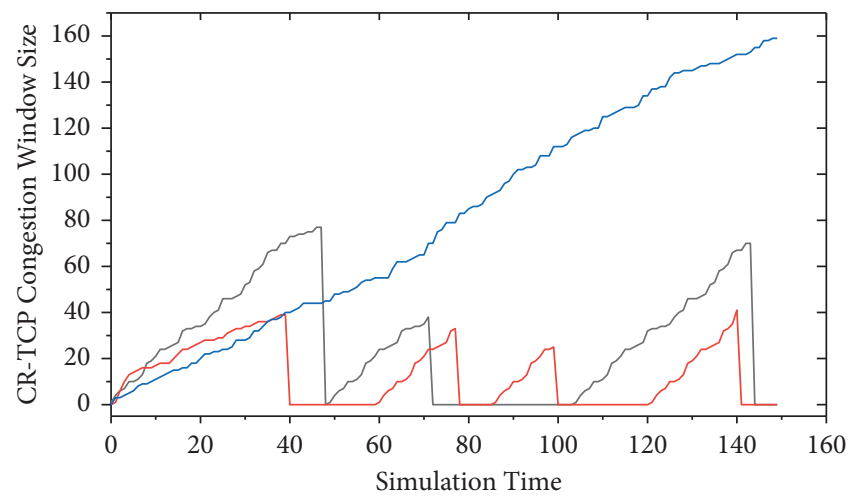

- CR-AODV without spectrum handover buffer

_ CR-AODV with only spectrum handover buffer

CR-AODV with spectrum handover buffer + priority bit

(a)

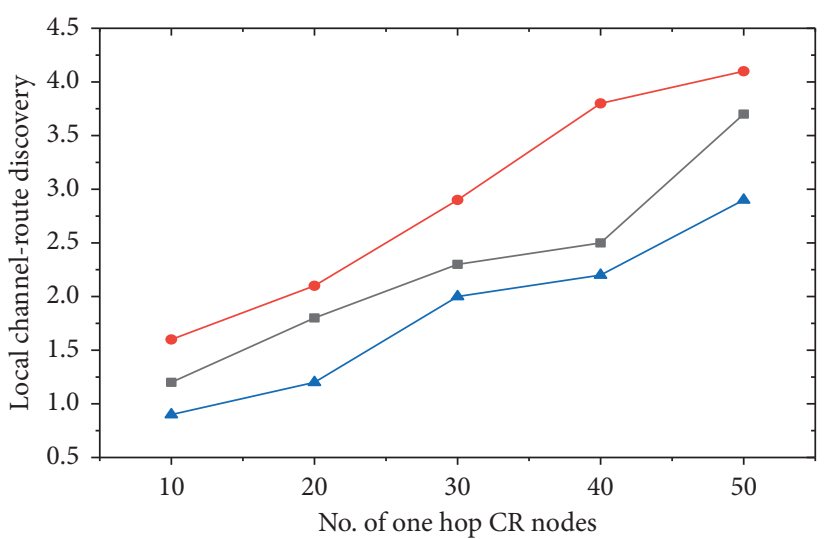

$\rightarrow$ CR-AODV with only spectrum handover buffer

$\rightarrow$ CR-AODV without spectrum handover buffer

^ CR-AODV with spectrum handover buffer + priority bit

(c)

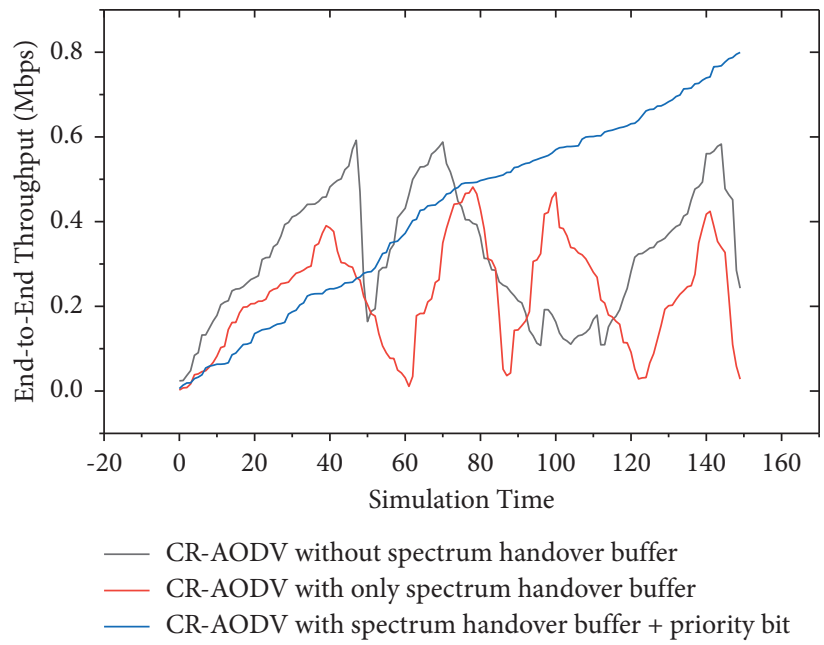

(b)

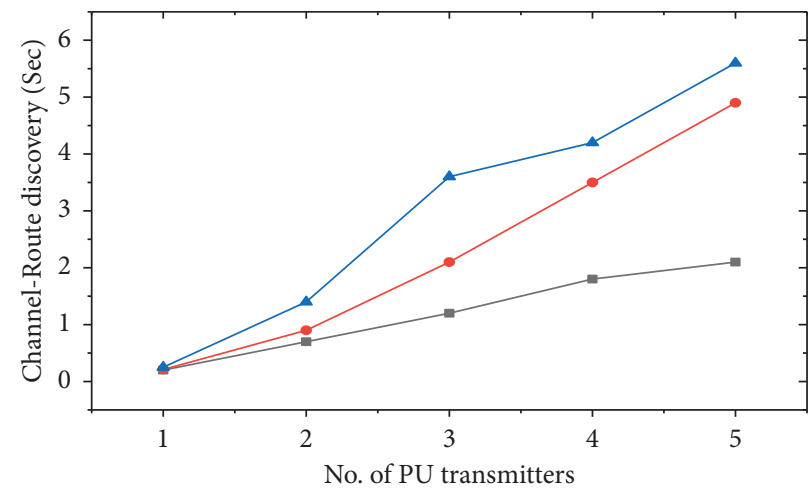

- CR-AODV with spectrum handover buffer + priority bit

- CR-AODV with only spectrum handover buffer

$\_$CR-AODV without spectrum handover buffer

Figure 5: Continued. 


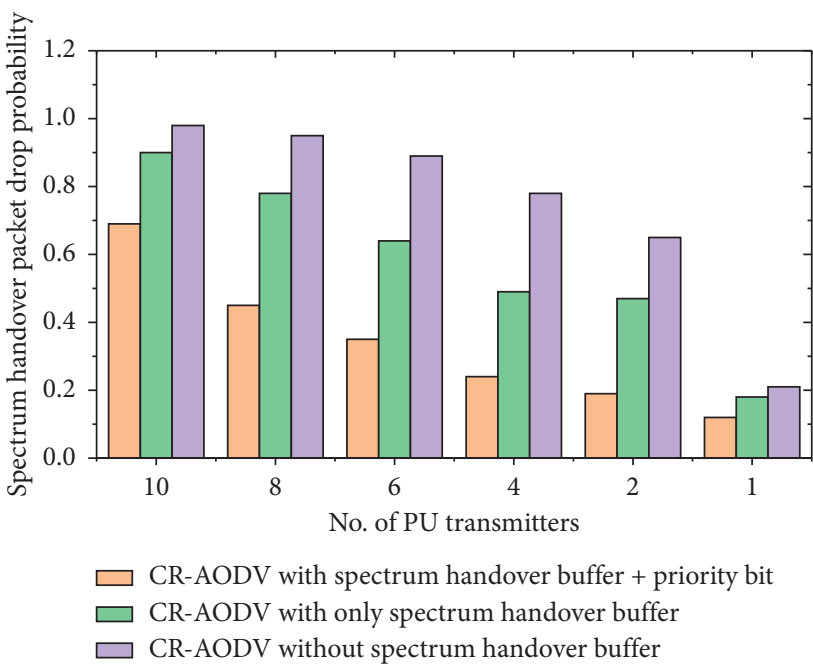

(e)

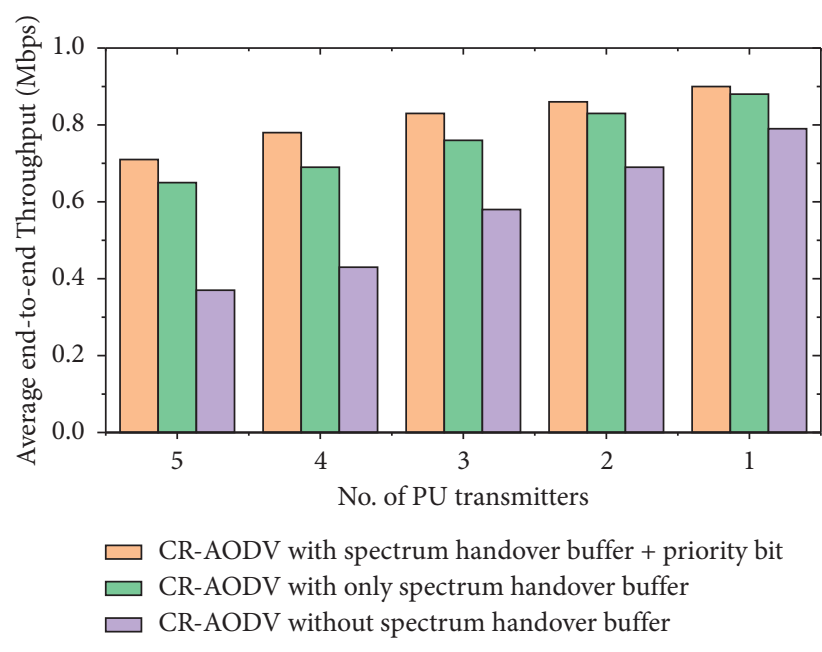

(f)

FIGURE 5: Cognitive TCP performance evaluation with respect to spectrum handoff. (a) CR-TCP congestion window variation with varied simulation time. (b) End-to-end TCP throughput with respect to simulation time. (c) Spectrum handoff local channel-route discovery with respect to no. of CR nodes. (d) Spectrum handoff local channel-route discovery with respect to the PU transmitters. (e) Spectrum handover packet drop probability with respect to no. of PU transmitters. (f) Average end-to-end CR-TCP throughput with respect to no. of PU transmitters.

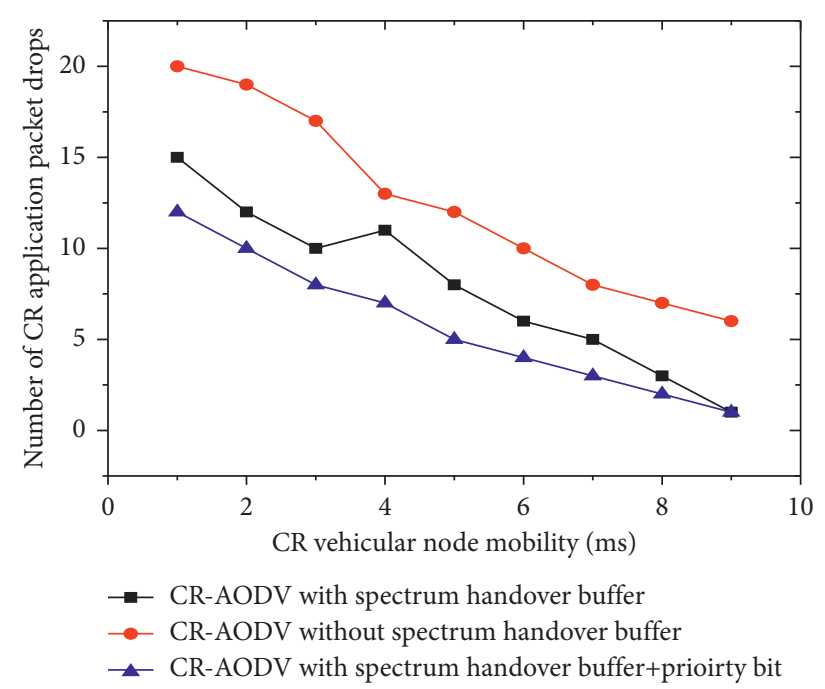

(a)

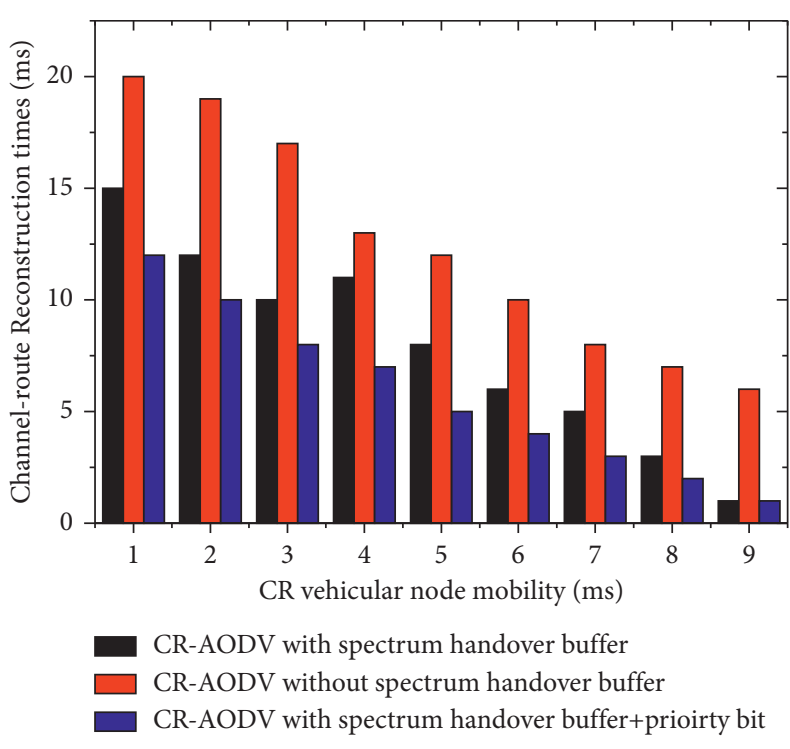

(b)

FIgURE 6: Performance of the CR vehicular network. (a) CR vehicular node mobility variation with respect to the number of application packet drops. (b) $\mathrm{CR}$ node mobility variation with respect to channel-route reconstruction time.

RED buffer will not drop the outgoing packets with priority enabled, whereas for the other two cases, there is more probability of packet drops at the output RED buffer. Figure 5(d) describes the local channel-route discovery with respect to the number of PU transmitters. In general, with increased PU transmitters, the probability of getting the opportunistic channel to transmit the CR application data gets reduced. In addition, the ongoing traffic flow gets packet drops because of spectrum handover at the intermediate CR nodes. Thus, the solutions with separate handover buffers at an intermediate CR node will attain better throughput in comparison with the solutions that does not support the packet buffers and priority bit. Figure 5(e) describes the probability of spectrum handover packet drops with respect to the number of PU transmitters. As shown in Figure 5(e), it is clear that the probability of the packet drop with respect to spectrum handover is directly proportional to the number of PU transmitters. Moreover, packet drop at the intermediate CR node gets dropped when there are no separate handover buffers. In other words, the input and output RED buffers 
cannot hold on-the-fly CR packets until a new local channel link gets discovered. Thus, the probability of the CR packet drop is higher with the solutions that do not support spectrum handover buffers. Figure 5(f) describes the average end-to-end CR-TCP throughput with respect to the number of PU transmitters. CR-AODV with spectrum handover buffer along with priority bit is having higher achievable average end-to-end throughput in comparison with the other two techniques. This is due to reduced on-the-fly packet drops and reduced network congestion CR packet drops at the intermediate CR node.

Figure 6 explains about the CR vehicular ad-hoc network performance with respect to $\mathrm{CR}$ node handover from one base station to another base station. In general, when an intermediate CR vehicular node transits from one coverage area to other coverage areas, then the in-flight forwarded packets have to be either stored in a buffer of aggregate traffic flow buffer or CR node mobility priority buffer. In our experimental results of Figure 6(a), we tested the performance of the CR vehicular adhoc network with different node handover mobility scenarios. As shown in Figure 6(a), the packets drops are high when the probability of node handover from one base station to another base station is very high. In other words, the packet drops with node mobility variation of $1 \mathrm{msec}$ are much higher than the packet drops with node mobility variation of $8 \mathrm{msec}$. This is due to heavy inflight traffic flow at the intermediate CR nodes at the time of CR node mobility. The CR node mobility variation with respect to channel reconstruction time is compared in Figure 6(b). It is noteworthy that when a CR node handover to another base station, then the opportunistic licensed channel needs to be tested before continuing with the data transmission. Otherwise, there may be a collision with the primary user data which needs to be protected with higher priority. In Figure 6(b), channel reconstruction times increase with frequent CR vehicular node handover in comparison with the nodes that hand over with longer delays. This is because when a CR node handovers from one base station to another base station more frequently, then inflight packets have to be buffered in more number of times at the base station that results in congestion with the aggregate traffic flows.

\section{Conclusion}

Performance of CR-TCP in CR-VANET directly depends on the number of CR packet drops in between the source and destination CR nodes. With opportunistic dynamic channel access, there is a new packet drop due to spectrum handover (PU active in the current $\mathrm{CR}$ communication channel) in between the source and destination CR nodes. To alleviate the spectrum handover packet drops, this paper proposes a "spectrum handover-based AODV routing protocol in CRAHNs." With the proposed packet buffering and forwarding mechanism, the achievable end-to-end average throughput is enhanced as compared to the existing CR-AODV routing protocols.

\section{Data Availability}

The data used to support the findings of the study are included within this article.

\section{Conflicts of Interest}

The authors declare that there are no conflicts of interest regarding the publication of this paper.

\section{Acknowledgments}

This work was supported by the Key Project of Sichuan Province Science and Technology (2021YFG0186).

\section{References}

[1] S. H. Chun and R. J. La, "Secondary spectrum trading-auction-based framework for spectrum allocation and profit sharing," IEEE/ACM Transactions on Networking, vol. 21, no. 1, 2013.

[2] C. Lăcătusş, D. Akopian, P. Yaddanapudi, and M. Shadaram, "Flexible spectrum and power allocation for OFDM unlicensed wireless systems," IEEE System Journal, vol. 3, no. 2, 2009.

[3] T. Baykas, M. Kasslin, M. Cummings et al., "Developing a standard for TV white space coexistence: technical challenges and solution approaches," IEEE Wireless Communications, vol. 19, no. 1, 2012.

[4] F. B. S. D. Carvalho, W. T. A. Lopes, M. S. Alencar, and J. V. S. Filho, "Cognitive vehicular networks: an overview," Procedia Computer Science, vol. 65, 2015.

[5] R. S. Aqeel and A. Y. Kok-Lim, "Spectrum leasing in cognitive radio networks: A survey," International Journal of Distributed Sensor Networks, vol. 10, p. 2, 2014.

[6] R. Tandra, S. M. Mishra, and A. Sahai, "What is a spectrum hole and what does it take to recognize one?" 2009.

[7] B. F. Lo, "A survey of common control channel design in cognitive radio networks," Physical Communication, vol. 4, no. 1, 2011.

[8] M. Cesana, F. Cuomo, and E. Ekici, "Routing in cognitive radio networks: challenges and solutions," Ad Hoc Networks, vol. 9, no. 3, pp. 228-248, 2011.

[9] uting Wang, G. Zheng, H. Ma, Li Yang, and J. Li, “A joint channel selection and routing protocol for cognitive radio network," Wireless Communications and Mobile Computing, vol. 2018, Article ID 6848641, 7 pages, 2018.

[10] H. Khalifé, N. Malouch, and S. Fdida, "Multihop cognitive radio networks: to route or not to route," IEEE Network, vol. 23, no. 4, 2009.

[11] N. Devroye, M. Vu, and V. Tarokh, "Cognitive radio networks: highlights of information theoretic limits, models, and design," IEEE Signal Processing Magazine, vol. 26, no. 7, 2008.

[12] A. Guirguis, F. Digham, K. G. Seddik, M. Ibrahim, K. A. Harras, and M. Youssef, "Primary user-aware optimal discovery routing for cognitive radio networks," IEEE Transactions on Mobile Computing, vol. 18, no. 1, pp. 193-206, 2019.

[13] S. W. Kim, M. Pan, G. P. Joshi, O. Gazi, J. He, and M. Coupechoux, "Applications of cognitive radio networks: recent advances and future directions," International Journal of Distributed Sensor Networks, vol. 12, p. 1, 2016.

[14] I. F. Akyildiz, W. Y. Lee, and K. R. Chowdhury, "Spectrum management in cognitive radio ad hoc networks," IEEE Network, vol. 23, no. 4, 2009.

[15] K. Kotobi, P. Mainwaring, C. Tucker, and S. Bilén, "Datathroughput enhancement using data mining-informed cognitive radio," Electronics, vol. 4, no. 2, 2015. 
[16] S. Anamalamudi and M. Jin, "Low rate common control channel based AODV routing protocol for cognitive radio adhoc networks," in Proceedings of the International Conference on Ubiquitous and Future Networks, ICUFN, Da Nang, Vietnam, July 2013.

[17] M. Zareei, A. K. M. Muzahidul Islam, S. Baharun, C. VargasRosales, L. Azpilicueta, and N. Mansoor, "Medium access control protocols for cognitive radio ad hoc networks: A survey," Sensors (Switzerland), vol. 17, no. 9, 2017.

[18] K. Singh and S. Moh, "An energy-efficient and robust multipath routing protocol for cognitive radio ad hoc networks," Sensors (Switzerland), vol. 17, no. 9, 2017.

[19] S. Anamalamudi and M. Jin, "Performance enhancement of TCP in cognitive mobile IP based networks," in Proceedings of the International Conference on Ubiquitous and Future Networks, ICUFN, Shanghai, China, July 2014.

[20] S. Anamalamudi and M. Jin, "Energy-efficient hybrid CCCbased MAC protocol for cognitive radio ad hoc networks," IEEE Systems Journal, vol. 10, no. 1, pp. 358-369, 2016.

[21] T. Safdar Malik and M. Hilmi Hasan, "Reinforcement learning-based routing protocol to minimize channel switching and interference for cognitive radio networks," Complexity, vol. 2020, Article ID 8257168, 24 pages, 2020.

[22] A. Iqbal, R. Hussain, A. Shakeel et al., "Enhanced spectrum access for QoS provisioning in multi-class cognitive D2D communication system," IEEE Access, vol. 9, pp. 3360833624, 2021.

[23] A. R. Sangi, M. S. Alkatheiri, S. Anamalamudi, and J. Liu, "Cognitive AODV routing protocol with novel channel-route failure detection," Multimedia Tools and Applications, vol. 79, 2019.

[24] S. Anamalamudi, M. Jin, J. M. Kim, and C. Liu, "On demand priority packet forwarding for TCP performance enhancement in cognitive mobile IP networks," Wireless Personal Communications, vol. 86, no. 4, 2016.

[25] K. N. Qureshi, A. H. Abdullah, M. Bukhari, and R. W. Anwar, "SSNM-Smart sensor network model for vehicular ad hoc networks," in Proceedings of the 2015 International Conference on Smart Sensors and Application (ICSSA), pp. 82-87, Kuala Lumpur, Malaysia, May 2015.

[26] K. N. Qureshi, S. Din, G. Jeon, and F. Piccialli, "Link quality and energy utilization based preferable next hop selection routing for wireless body area networks," Computer Communications, vol. 149, pp. 382-392, 2020.

[27] K. N. Qureshi, A. H. Abdullah, O. Kaiwartya, U. Fasee, I. Saleem, and A. Ayman, "Weighted link quality and forward progress coupled with modified RTS/CTS for beaconless packet forwarding protocol (B-PFP) in VANETs," Telecommunication Systems, vol. 75, 2016.

[28] K. N. Qureshi, A. H. Abdullah, J. Lloret, and A. Altameem, "OBPF: opportunistic beaconless packet forwarding strategy for vehicular ad hoc networks," KSII Transactions on Internet and Information Systems, vol. 10, no. 5, pp. 2144-2165, 2016.

[29] A. Meddeb Makhlouf and M. Guizani, "SE-AOMDV: Secure and efficient AOMDV routing protocol for vehicular communications," International Journal of Information Security, vol. 18, no. 5, pp. 665-676, 2019.

[30] A. Ali, L. Abbas, M. Shafiq et al., "Hybrid fuzzy logic scheme for efficient channel utilization in cognitive radio networks," IEEE Access, vol. 7, pp. 24463-24476, 2019.

[31] Z. Ye, Y. Wang, and P. Wan, "Joint channel allocation and power control based on long short-term memory deep Q network in cognitive radio networks," Complexity, vol. 2020, Article ID 1628023, 11 pages, 2020.
[32] C. Cormio and K. R. Chowdhury, "Common control channel design for cognitive radio wireless ad hoc networks using adaptive frequency hopping," Ad Hoc Networks, vol. 8, no. 4, 2010.

[33] A. Misra and S. Banerjee, "MRPC: Maximizing network lifetime for reliable routing in wireless environments," in Proceedings of the 2002 IEEE Wireless Communications and Networking Conference Record. WCNC 2002 (Cat. No.02TH8609), Orlando, FL, USA, March 2002.

[34] Q. Li, M. Yang, H. Wang, Y. Jiang, and J. Zeng, "A finite queue model analysis of PMRC-based wireless sensor networks," in Proceedings of the 2008 International Conference on Wireless Networks, ICWN 2008, pp. 30-35, Las Vegas, NV, USA, July 2008.

[35] J Zhong, "Development of NS-2 based cognitive radio cognitive network simulator," M. S. thesis, Michigan Technological University, Houghton, MI, USA, 2009.

[36] C. Cordeiro, K. Challapali, D. Birru, and N. Sai Shankar, "IEEE 802.22: The First worldwide wireless standard based on cognitive radios," in Proceedings of the 2005 1st IEEE International Symposium on New Frontiers in Dynamic Spectrum Access Networks, DySPAN 2005, Baltimore, MD, USA, November 2005.

[37] M. Nekovee, "A survey of cognitive radio access to TV white spaces," in Proceedings of the International Journal of Data Mining and Bioinformatics, St. Petersburg, Russia, October 2010. 